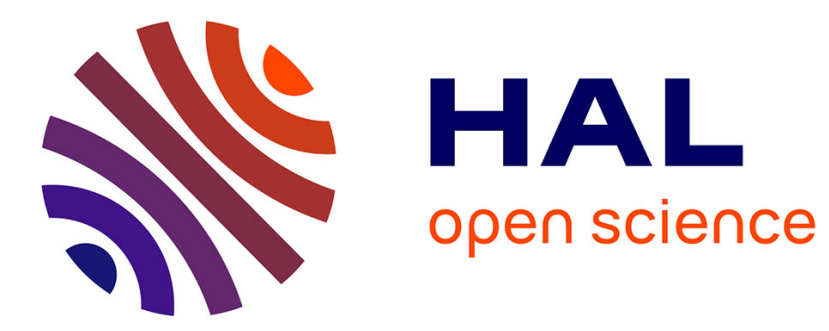

\title{
Two-component versus three-component metasolids
}

Elie Favier, Navid Nemati, Camille Perrot

\section{To cite this version:}

Elie Favier, Navid Nemati, Camille Perrot. Two-component versus three-component metasolids. Journal of the Acoustical Society of America, 2020, 148 (5), pp.3065 - 3074. 10.1121/10.0002424 . hal$03059349 v 2$

\section{HAL Id: hal-03059349 \\ https://hal.science/hal-03059349v2}

Submitted on 15 Mar 2021

HAL is a multi-disciplinary open access archive for the deposit and dissemination of scientific research documents, whether they are published or not. The documents may come from teaching and research institutions in France or abroad, or from public or private research centers.
L'archive ouverte pluridisciplinaire HAL, est destinée au dépôt et à la diffusion de documents scientifiques de niveau recherche, publiés ou non, émanant des établissements d'enseignement et de recherche français ou étrangers, des laboratoires publics ou privés. 


\title{
Two-component versus three-component metasolids
}

\author{
Elie Favier, Navid Nemati, ${ }^{\text {a) }}$ and Camille Perrot \\ MSME, Univ Gustave Eiffel, CNRS UMR 8208, Univ Paris Est Creteil, F-77454 \\ Marne-la-Vallée, France
}

(Dated: 7 October 2020)

Analytic analysis and parametric investigation are employed to study and compare metamaterial properties of two types of composite metasolids. Metasolids are composed of either an elastic inclusion or a rigid core coated by an elastic material, embedded in a stiff matrix. For these types of materials, results related to cylindrical as well as spherical inclusions are presented. Such monoinclusion two-component and bi-inclusion three-component metasolids have been previously known to exhibit negative mass density near local-resonance frequencies. Through a unified formulation, it is analytically shown how and why adding a rigid mass inside the elastic inclusion to make a bi-inclusion threecomponent material, can dramatically change the homogenized property of the resultant inclusion and increase the tunability of the composite, particularly, in terms of local-resonance frequencies and the associated metamaterial-effect frequency bandwidth. In this way, concerning distinctly sound and vibration insulation, low-frequency metamaterial effect with larger bandwidth can be designed via an inverse problem using a simplified mass-spring model.

(C)2020 Acoustical Society of America. [http://dx.doi.org(DOI number)]

$[\mathrm{XYZ}]$

Pages: 1-13

\section{INTRODUCTION}

Elastic metamaterials or metasolids are composite structures with subwavelength units that exhibit extreme frequency-dependent macroscopic parameters. In particular, the first metasolid has been designed through locally resonant structural units made of high-density and stiff lead sphere coated with a soft material that are embedded in a stiff material ${ }^{1}$. This threecomponent composite has been shown by an analytic model to produce negative mass density near local-resonance frequencies ${ }^{2}$. The model is based on the assumption that the solid core as well as the host medium are rigid and only the soft cladding is elastic. The first step to perform homogenization according to this scheme is to obtain the effective mass density $\rho_{e}$ of the entire inclusion that includes the hard lead (medium 3) and the soft coating (medium 2). To do so, dynamic equilibrium is considered between the ho-

a)navid.nemati@u-pem.fr

J. Acoust. Soc. Am. / 7 October 2020 mogenized medium (core+cladding) which undergoes an acceleration of $-\omega^{2} \boldsymbol{U}_{\boldsymbol{b}}$, e.g., in the direction $\boldsymbol{e}_{x}$ for the cylinder or $\boldsymbol{e}_{z}$ for the sphere (Fig. 1), and the resultant of the contact forces on the interface of the matrix and the inclusion. This is translated into the following equation

$$
-\omega^{2} \rho_{e}^{3 c}(\omega) V_{e} \boldsymbol{U}_{\boldsymbol{b}}=\int_{\mathcal{S}} \boldsymbol{\sigma} \cdot \boldsymbol{n} d S
$$

where $V_{e}$ is the volume of the entire inclusion, $\boldsymbol{n}$ is the normal vector outward from the surface of the medium 2 to the medium 1 , and the integration is performed over the surface of the inclusion $\mathcal{S}$, that is the interface between the medium 1 and medium 2. The superscript $3 c$ is used to refer to three-component materials. The stress tensor in the elastic medium $2, \boldsymbol{\sigma}$, is related to the displacement field $\boldsymbol{u}$ in the same medium, via $\boldsymbol{\sigma}=\mu_{2}\left(\boldsymbol{\nabla} \boldsymbol{u}+\boldsymbol{\nabla} \boldsymbol{u}^{T}\right)+\lambda_{2}(\boldsymbol{\nabla} \cdot \boldsymbol{u}) \mathcal{I}$, with $\boldsymbol{u}^{T}$ the transpose of $\boldsymbol{u}, \mathcal{I}$ the identity matrix; and $\lambda_{2}$ and $\mu_{2}$ the Lamé parameters. The effective parameter $\rho_{e}^{3 c}$ can be calculated, once the stress tensor and, thereby, the microscopic displacement field in medium 2 is determined. This can be achieved 


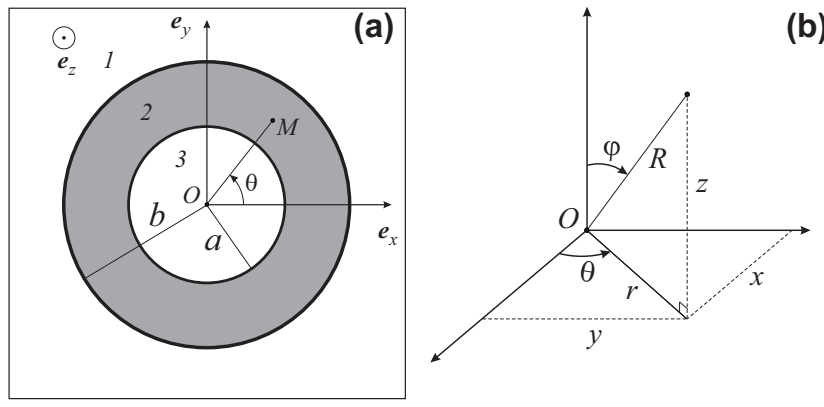

FIG. 1. Illustration of a unit cell with cylindrical or spherical inclusion in $x y$ plane (a), and coordinate system in $3 \mathrm{D}$ space (b). The position vector $\boldsymbol{O} \boldsymbol{M}=$ $r \boldsymbol{e}_{r}+z \boldsymbol{e}_{z}$ in media with cylindrical inclusions $(2 \mathrm{D}$ system), and $\boldsymbol{O} \boldsymbol{M}=\boldsymbol{R} \boldsymbol{e}_{R}$ in media with spherical inclusions (3D system). The gradient operator in $2 \mathrm{D}$ system is written as $\boldsymbol{\nabla}=\boldsymbol{e}_{r} \frac{\partial}{\partial r}+\boldsymbol{e}_{\theta} \frac{1}{r} \frac{\partial}{\partial \theta}+\boldsymbol{e}_{z} \frac{\partial}{\partial z}$, and in $3 \mathrm{D}$ system: $\boldsymbol{\nabla}=\boldsymbol{e}_{R} \frac{\partial}{\partial R}+\boldsymbol{e}_{\varphi} \frac{1}{R} \frac{\partial}{\partial \varphi}+\boldsymbol{e}_{\theta} \frac{1}{R \sin \varphi} \frac{\partial}{\partial \theta}$.

by solving the microscopic elastodynamic equation of motion in the coating material. The second and final step is to specify the effective mass density of the whole structural unit, including the matrix, through a simple mixing law

$$
\rho_{\text {eff }}^{3 c}(\omega)=\left(\phi_{2}+\phi_{3}\right) \rho_{e}^{3 c}(\omega)+\phi_{1} \rho_{1},
$$

where $\phi_{1}, \phi_{2}$ and $\phi_{3}$ are the filling fraction of the matrix, the cladding and the rigid core, respectively; such that $\phi_{1}+\phi_{2}+\phi_{3}=1$. In our previous paper, the same model and homogenization method was employed in order to study the rotational modes ${ }^{3}$.

A commonly used technique for determining effective properties of metasolids is based on Plane Wave Expansions (PWE) of fields and Fourier space representation of material properties ${ }^{4,5}$. Recent homogeniezation development ${ }^{6}$ employing PWE involves space nonlocality $^{7,8}$ according to Willis viewpoint of constitutive equations ${ }^{9}$. Another broad effective medium method for homogenization to capture local resonances in metasolids is through Coherent Potential Approximation $(\mathrm{CPA})^{10}$ based on multipole expansion minimizing scatterings in the long-wavelength limit ${ }^{11,12}$. With similar ideas, an enhanced scheme has been developed to obtain the effective properties of the same type of materials in a wider frequency band by matching the lowest-order scattering amplitudes that arise from the unit cell, with that of the homogenized material ${ }^{13}$. By multipole expansion, scattered waves from structural units can be displayed by superposition of modes with different angular momenta. Within this representation, effective mass density can be associated with the dipole term and bulk modulus has the monopole symmetry. Although multipole method is less flexible than PWE method in terms of the geometry of the structural unit, it may be more appropriate to obtain analytic results regarding effective-medium parameters.

Recently, asymptotic homogenization methods has been developed to describe the dynamics of a two-component composite material made of spherical or cylindrical soft inclusions embedded in a stiff matrix ${ }^{14,15}$. In this paper, it will be shown that these results at microscopic scale can be obtained as the particular case of elastodynamic analytical results from three-component materials. To do so, the analytic model that has been previously studied ${ }^{2,3}$ and elements of which was mentioned above, is briefly recalled and then employed to show that existing results for two-component materials can be derived from those for three-component materials in the limit the geometrical form factor $\gamma \equiv b / a \gg 1$ (Fig. 1a). It will be then demonstrated that, within this geometrical limit, at macroscopic scale, where the material is homogenized, the analytical expressions of the frequency-dependent effective densities of the two-component materials results from those of the three-component ones. As such, using this approach, the dynamics of the two and three component materials are explicitly described through a unified and coherent formulation, and the difference between the physics emerged from these material types can be grasped. The comparative analysis is carried out for materials with spherical inclusions constituting a three-dimensional (3D) system, as well as with cylindrical inclusions in the form of a two-dimensional (2D) system.

Also, the systematic comparison of the twocomponent and the three-component materials that is presented in this paper, highlights the practical advantages of the latter compared to the former. In particular, it will be shown that contrary to the three component materials, the desired elastic properties for the inclusion of twocomponent materials, if not impossible, are difficult to obtain with a single elastic medium. 
It is now well known that local resonances can create metamaterial phenomena at macroscale; in this case, negative effective mass density near the resonance frequencies, where bandgaps appear. By comparing the twocomponent and the three-component materials, it will be also clarified how the three-component materials can produce a macroscopic dynamic response richer than that of the two-component ones; including in particular the generation of a low-frequency resonance that is associated with the motion of the rigid core. This resonance phenomenon may be more interesting for applications such as sound and vibration insulation, because it can occur at lower frequencies and, at the same time, with a possible wider frequency band. It will be explained that localresonance frequencies are relatively high with two-component materials, because even in the case where the elasticity coefficients related to the matrix and the inclusion are sufficiently contrasted, the contrast may not exist for the mass densities; and vice versa. With such materials, it is indeed very difficult to find, in practice, a low rigidity and, at the same time, a high mass density for the elastic material used as inclusion inside the stiff matrix. It will be shown that this could be feasible by dissociating these two material properties, i.e. softness for the cladding and mass density for the coated rigid core, with a three-component material whose inclusion is composed of two phases.

Additionally, the discrete mass-spring model ${ }^{3,16,17}$ that was validated in Ref. ${ }^{3}$ allows the quick construction of analytical analysis in terms of determination of the first (lowest) resonance frequency as well as estimation of the bandgap width created by the material local resonance. This aspect will be demonstrated in the paper, through a parametric study. The method facilitates the design of the structure via solving the inverse problem, and also makes it possible to properly optimize the acoustic properties of such a structure, with respect to an application of interest; particularly in terms of the resonance frequency and the frequency bandwidth where the metamaterial effect occurs. Indeed, the mass-spring model enables to find the micro-structural characteristics (inverse problem) in order to then produce via the elasto-dynamic model a metasolid with the targeted macro-structural properties (direct problem). Thus, in brief, the goal of this paper is to compare the homogenized dynamic properties of two-component and three-component materials in terms of firstly, origin and physical modeling, and secondly, parametric study. The latter leads to the formulation of an inverse problem that allows the design of a metasolid with the desired properties.

In the following section, the results arising from analytic modeling based on microscopic elastodynamic equations, related to threecomponent and two-component metamaterials both in 2D and 3D systems, are presented and compared, followed by the comparative analysis of effective mass densities based on analytic modeling at macroscale, associated with these two material types. In Sec. III, the analytic results and their consequences are illustrated through an example and a parametric study is presented before its application to clarify a method to design optimal structures in Sec. IV. Concluding remarks are given in Sec. IV.

\section{COMPARISON OF TWO-COMPONENT AND THREE-COMPONENT MATERIALS}

Concerning two-component metasolids, based on an asymptotic method, it has been demonstrated in Ref. ${ }^{14}$ that, in the longwavelength regime, where $\epsilon \equiv \ell / L \ll 1$, with $\ell$, being the size of the structural unit and $L$, the order of the magnitude of the wavelength of the wave propagating in the material; the local resonances occur under certain conditions with respect to microscopic material properties. In particular, the mass densities of the matrix and the inclusion must be of the same order of magnitude, while the ratio of the bulk moduli of the matrix and the inclusion must be of the order $O\left(\epsilon^{2}\right)^{18-20}$; that means the stiffnesses of matrix and the inclusion should be highly contrasted. In other words, the mass density of the inclusion must be close to that of the matrix while being much softer than the matrix. Indeed, practically in terms of choice of the materials in a two-component composite, it is not easy that these two conditions are satisfied simultaneously.

Here, it is investigated if the results from twocomponent material can be found as the limit of those from the three-component material. To do so, wherever the explicite results related to twocomponent material are available in Refs. ${ }^{14,15}$, 
in particular, concerning the microscopic displacement fields in the monophasic elastic inclusion as well as the material local-resonance frequencies, a comparison is carried out between them and those arising from taking the limit of the dynamics of three-component materials. It is expected that the three-component material is transitioned to the two-component material when the diameter of the lead core $a$ becomes much smaller than the diameter of the whole inclusion $b$ (Fig. 1a). This limit can be expressed in terms of the form factor $\gamma=b / a$, as $\gamma \gg 1$.

\section{A. Microscopic description}

In thee-component material, the equation of motion in the elastic coating is written as

$\left(\lambda_{2}+2 \mu_{2}\right) \boldsymbol{\nabla}(\boldsymbol{\nabla} \cdot \boldsymbol{u})-\mu_{2} \boldsymbol{\nabla} \times(\boldsymbol{\nabla} \times \boldsymbol{u})=-\rho_{2} \omega^{2} \boldsymbol{u}$

where $\rho_{2}$ is the mass density of medium 2. The displacement fields can be calculated from two potential fields $\Phi$ and $\Psi$, defined such that ${ }^{2,3}$

$$
\boldsymbol{u}=\boldsymbol{\nabla} \Phi+\boldsymbol{\nabla} \times \boldsymbol{\Psi}, \quad(\boldsymbol{\nabla} \cdot \boldsymbol{\Psi}=0)
$$

which are solutions to the following Helmholtz equations, when the relation (4) is substituted into the equation (3):

$$
\begin{aligned}
\nabla^{2} \Phi+h^{2} \Phi & =0 \\
\nabla^{2} \boldsymbol{\Psi}+\kappa^{2} \boldsymbol{\Psi} & =\mathbf{0} .
\end{aligned}
$$

In the above equations, $h=\omega \sqrt{\rho_{2} /\left(\lambda_{2}+2 \mu_{2}\right)}$ and $\kappa=\omega \sqrt{\rho_{2} / \mu_{2}}$ are the wavenumbers associated with the longitudinal and transverse elastic waves, respectively. In the following, analytic solutions shall be provided to Helmholtz equations (5) with the displacement-continuity boundary conditions, for two special cases of major interest. It is convenient to use the coordinate system shown in Fig. 1 for both cylindrical and spherical coordinates, applied for materials with cylindrical and spherical inclusions, respectively; as these materials are studied in the paper, in parallel. Thus, notice that in the coordinate system of Fig. 1b, the angle $\theta$ is used in an unusual way in spherical coordinates with its operator $\nabla$ defined in the figure's caption; also the position vector and its associated unit vector are denoted by $\boldsymbol{R}$ and $\boldsymbol{e}_{R}$, respectively. Owing to their symmetry property, materials with cylindrical inclusions are only studied in a cross-sectional plane perpendicular to their symmetry axis; here, in xy plane. That is why they will be often called as $2 \mathrm{D}$ systems in the paper. The boundary conditions for the displacement field in 2D system are written as $U_{\alpha} \boldsymbol{e}_{x}, \alpha=a, b$, at the surfaces $r=\alpha$. This can be expressed according to cylindrical coordinates as

$$
\left.\boldsymbol{u}\right|_{r=\alpha}=U_{\alpha}\left(\cos \theta \boldsymbol{e}_{r}-\sin \theta \boldsymbol{e}_{\theta}\right) .
$$

For the 3D systems, the boundary conditions are $U_{\alpha} \boldsymbol{e}_{z}$ at the surfaces $R=\alpha, \alpha=a, b$, which becomes

$$
\left.\boldsymbol{u}\right|_{R=\alpha}=U_{\alpha}\left(\cos \varphi \boldsymbol{e}_{R}-\sin \varphi \boldsymbol{e}_{\varphi}\right),
$$

in spherical coordinate system.

The form of the solutions to the Helmholtz boundary value problems are given in Appendix A, involving the unknown constants $\left\{A_{1}, B_{1}, E_{1}, F_{1}\right\}$ for $2 \mathrm{D}$ system, and $\left\{a_{1}, b_{1}, e_{1}, f_{1}\right\}$ for $3 \mathrm{D}$ system, to be determined by using the boundary relations at the interfaces of each two phases in the structural unit.

The components of the displacement field are found, according to cylindrical coordinates, for the $2 \mathrm{D}$ system as

$$
u_{r}=\frac{\partial \Phi}{\partial r}+\frac{1}{r} \frac{\partial \Psi_{z}}{\partial \theta}=L(r) \cos \theta
$$

with

$$
\begin{aligned}
L(r)= & \frac{1}{r}\left[h r\left(A_{1} \mathrm{~J}_{1}^{\prime}(h r)+B_{1} \mathrm{Y}_{1}^{\prime}(h r)\right)\right. \\
& \left.+E_{1} \mathrm{~J}_{1}(\kappa r)+F_{1} \mathrm{Y}_{1}(\kappa r)\right] \\
u_{\theta}= & \frac{1}{r} \frac{\partial \Phi}{\partial \theta}-\frac{\partial \Psi_{z}}{\partial r}=-M(r) \sin \theta
\end{aligned}
$$

with

$$
\begin{aligned}
M(r)= & \frac{1}{r}\left[A_{1} \mathrm{~J}_{1}(h r)+B_{1} \mathrm{Y}_{1}(h r)\right. \\
& \left.+\kappa r\left(E_{1} \mathrm{~J}_{1}^{\prime}(\kappa r)+F_{1} \mathrm{Y}_{1}^{\prime}(\kappa r)\right)\right]
\end{aligned}
$$

In the above equations and in the following, the derivatives of the Bessel functions are kept, but it is known that these derivatives can be easily expressed in terms of the Bessel functions, e.g., $x Z_{1}^{\prime}(x)=x Z_{0}(x)-Z_{1}(x)=Z_{1}(x)-x Z_{2}(x)$.

The components of the displacement field for 3D system, according to spherical coordinates are expressed as:

$$
u_{R}=\frac{\partial \Phi}{\partial R}+\frac{1}{R \sin \varphi} \frac{\partial}{\partial \varphi}\left(\sin \varphi \Psi_{\theta}\right)=l(R) \cos \varphi
$$


with

$$
\begin{gathered}
l(R)=\frac{1}{R}\left[h R\left(a_{1} \mathrm{j}_{1}^{\prime}(h R)+b_{1} \mathrm{y}_{1}^{\prime}(h R)\right)\right. \\
\left.+2\left(e_{1} \mathrm{j}_{1}(\kappa R)+f_{1} \mathrm{y}_{1}(\kappa R)\right)\right] \\
u_{\varphi}=\frac{1}{R} \frac{\partial \Phi}{\partial \varphi}-\frac{1}{R} \frac{\partial}{\partial R}\left(R \Psi_{\theta}\right)=-m(R) \sin \varphi
\end{gathered}
$$

with

$$
\begin{aligned}
m(R)= & \frac{1}{R}\left[a_{1} \mathrm{j}_{1}(h R)+b_{1} \mathrm{y}_{1}(h R)+e_{1}\left(\mathrm{j}_{1}(\kappa R)\right.\right. \\
& \left.\left.+\kappa R \mathrm{j}_{1}^{\prime}(\kappa R)\right)+f_{1}\left(\mathrm{y}_{1}(\kappa R)+\kappa R \mathrm{y}_{1}^{\prime}(\kappa R)\right)\right] .
\end{aligned}
$$

As before in the above, the derivatives of the spherical Bessel functions can be expressed in terms of the Bessel functions, e.g., $x z_{1}^{\prime}(x)=$ $x z_{0}(x)-2 z_{1}(x)=z_{1}(x)-x z_{2}(x)$.

By using the relations associated with the boundary conditions in 2D materials, i.e. $L(\alpha)=$ $U_{\alpha}=M(\alpha)$, and 3D materials, i.e. $l(\alpha)=U_{\alpha}=$ $m(\alpha)$, with $\alpha=a, b$; it is easy to deduce the linear algebraic systems allowing the complete determination of the constants, that are involved in the solutions to Helmholtz equations and subsequently in the above expressions of the displacements fields in 2D and 3D systems (Appendix B).

Finally, the resonance frequencies is obtained by setting the determinants of the matrix $D$ [see Eq. (B1)] and the matrix $d$ [see Eq. (B2)] in Appendix B, equal to zero, regarding 2D system and 3D system, respectively. It is noted that these resonance frequencies are related to medium local-resonances, that occur in the soft cladding.

To find exactly the corresponding expressions of (A2) and (A3) solutions to Helmhotz equations (5) in Appendix A, for the case of two-component materials as in Ref. ${ }^{14}$, it is only needed to make disappear the functions $\mathrm{Y}_{1}$ and $\mathrm{y}_{1}$ in these equations. Indeed, given that the displacements fields must be finite at the center of the unit cell, when the rigid core disappears, in two-component materials, following the same procedure there will be only two unknown constants to be determined; instead of four constants involved in the equations (A2) and (A3) in Appendix A for the three-component materials. As such, in the case of two-component materials, the order of the equations to be solved and the associated matrices [Eqs. (B1) and (B2) in
Appendix B] are reduced, and thereby it is easier to obtain explicit analytic expressions for the displacement fields. It is also to be noted that in two-component materials the interfaces between different phases are reduced to only one interface $(\gamma=b / a \gg 1)$, which results in only one boundary condition inside the structural unit. When $\gamma \gg 1$, the following expressions are obtained for the $2 \mathrm{D}$ system:

$$
\begin{aligned}
& L(r)=-\frac{h \kappa b^{2} U_{b}}{\Delta_{2 D}}\left[\mathrm{~J}_{1}^{\prime}(h r) \mathrm{J}_{2}(\kappa b)+\frac{\mathrm{J}_{1}(\kappa r)}{\kappa r} \mathrm{~J}_{2}(h b)\right] \\
& M(r)=-\frac{h \kappa b^{2} U_{b}}{\Delta_{2 D}}\left[\frac{\mathrm{J}_{1}(h r)}{h r} \mathrm{~J}_{2}(\kappa b)+\mathrm{J}_{1}^{\prime}(\kappa r) \mathrm{J}_{2}(h b)\right]
\end{aligned}
$$

where

$$
\Delta_{2 D}=-\frac{h \kappa b^{2}}{2}\left[\mathrm{~J}_{0}(h b) \mathrm{J}_{2}(\kappa b)+\mathrm{J}_{0}(\kappa b) \mathrm{J}_{2}(h b)\right] ;
$$

and for the 3D system,

$$
\begin{aligned}
l(R) & =-\frac{h \kappa b^{2} U_{b}}{\Delta_{3 D}}\left[\mathrm{j}_{1}^{\prime}(h R) \mathrm{j}_{2}(\kappa b)+\frac{2 \mathrm{j}_{1}(\kappa R)}{\kappa R} \mathrm{j}_{2}(h b)\right] \\
m(R) & =-\frac{h \kappa b^{2} U_{b}}{\Delta_{3 D}}\left[\frac{\mathrm{j}_{1}(h R)}{h R} \mathrm{j}_{2}(\kappa b)+\frac{\left(x \mathrm{j}_{1}\right)^{\prime}(\kappa R)}{\kappa R} \mathrm{j}_{2}(h b)\right]
\end{aligned}
$$

where

$$
\Delta_{3 D}=-\frac{h \kappa b^{2}}{3}\left[\mathrm{j}_{0}(h b) \mathrm{j}_{2}(\kappa b)+2 \mathrm{j}_{0}(\kappa b) \mathrm{j}_{2}(h b)\right] .
$$

For materials with cylindrical inclusions, it should be pointed out that the above expressions for $L(r)$ and $M(r)$ are used in Eqs. (8) and (9) to describe the translational displacement fields inside the inclusion, only in the $x y$ plane (Fig. $1)$.

\section{B. Macroscopic description}

As it was explained earlier in Sec. I, the first step of the homogenization approach for threecomponent material consists of finding the effective mass density of the core and the cladding, i.e. $\rho_{e}$, through Eq. (1). After, taking into account of the equation of motion for the core material and finding the microscopic displacement field, the following results are obtained for the 2D system

$$
\begin{aligned}
& \rho_{e}^{3 c}(\omega)=\frac{-1}{\pi \omega^{2} b U_{b}} \int_{0}^{2 \pi}\left(\sigma_{r r} \cos \theta-\sigma_{r \theta} \sin \theta\right) d \theta \\
& =\frac{\rho_{2}}{b U_{b}}\left[A_{1} \mathrm{~J}_{1}(h b)+B_{1} \mathrm{Y}_{1}(h b)+E_{1} \mathrm{~J}_{1}(\kappa b)+F_{1} \mathrm{Y}_{1}(\kappa b)\right],
\end{aligned}
$$


and for the 3D system:

$\rho_{e}^{3 c}(\omega)=\frac{-3}{2 \omega^{2} b U_{b}} \int_{0}^{\pi}\left(\sigma_{R R} \cos \varphi-\sigma_{R \varphi} \sin \varphi\right) \sin \varphi d \varphi$

$=\frac{\rho_{2}}{b U_{b}}\left[a_{1} \mathrm{j}_{1}(h b)+b_{1} \mathrm{y}_{1}(h b)+2 e_{1} \mathrm{j}_{1}(\kappa b)+2 f_{1} \mathrm{y}_{1}(\kappa b)\right]$.

As it was mentioned before, the ensembles of constants $\left(A_{1}, B_{1}, E_{1}, F_{1}\right)$, and $\left(a_{1}, b_{1}, e_{1}, f_{1}\right)$, involved in the above equations, are known through solving the equations (B1) and (B2), respectively. Then, for both $2 \mathrm{D}$ and $3 \mathrm{D}$ systems, it is immediate to determine the effective mass density of the whole structure by the simple mixing law (2).

Applying the limit $\gamma \gg 1$, leads to the following explicit expressions that are related to two-component materials. For the 2D system, it reads

$\rho_{e}^{2 c}(\omega)=\rho_{2}\left\{1+\frac{2 \mathrm{~J}_{2}(h b) \mathrm{J}_{2}(\kappa b)}{\mathrm{J}_{0}(h b) \mathrm{J}_{2}(\kappa b)+\mathrm{J}_{0}(\kappa b) \mathrm{J}_{2}(h b)}\right\}$

and for 3D system:

$\rho_{e}^{2 c}(\omega)=\rho_{2}\left\{1+\frac{3 \mathrm{j}_{2}(h b) \mathrm{j}_{2}(\kappa b)}{\mathrm{j}_{0}(h b) \mathrm{j}_{2}(\kappa b)+2 \mathrm{j}_{0}(\kappa b) \mathrm{j}_{2}(h b)}\right\}$

where the superscript $2 c$ refers to twocomponent materials.

As it is expected, in the static regime, when $\omega \rightarrow 0$, the effective mass density of the biphasic inclusion $\rho_{e}^{3 c}(0)=\left[1 /\left(1-\phi_{1}\right)\right]\left(\phi_{2} \rho_{2}+\phi_{3} \rho_{3}\right)$, and that of the monophasic inclusion $\rho_{e}^{2 c}(0)=\rho_{2}$. Indeed, in the static regime, it is clear that $\rho_{0}^{3 c} \equiv \rho_{\text {eff }}^{3 c}(0)=\phi_{1} \rho_{1}+\phi_{2} \rho_{2}+\phi_{3} \rho_{3}$ and $\rho_{0}^{2 c} \equiv$ $\rho_{\text {eff }}^{2 c}(0)=\phi_{1} \rho_{1}+\left(1-\phi_{1}\right) \rho_{2}$. Regarding the relation (2), obviously, in the absence of the rigid mass (medium 3) the frequency-dependent effective mass density of the whole structure of the two-component material reads

$$
\rho_{e f f}^{2 c}(\omega)=\phi_{1} \rho_{1}+\left(1-\phi_{1}\right) \rho_{e}^{2 c}(\omega) .
$$

\section{MATERIAL EXAMPLE AND PARAMETRIC STUDY}

Here, we illustrate the analytical results of the previous section, through taking an example of the three-component as well as the twocomponent materials both with spherical inclusions, and with the appropriate materialproperty and geometrical similarities allowing
TABLE I. Material properties of the threecomponent unit-cell.

\begin{tabular}{cccc}
\hline \hline Material & $\begin{array}{c}\text { Alumina }^{\mathrm{a}} \\
(\text { matrix })\end{array}$ & $\begin{array}{c}\text { Polystyrene } \\
(\text { cladding })\end{array}$ & $\begin{array}{c}\text { Lead } \\
(\text { core })\end{array}$ \\
$\lambda[\mathrm{Pa}]$ & $1.4 \times 10^{8}$ & $2.54 \times 10^{5}$ & $4.23 \times 10^{10}$ \\
$\mu[\mathrm{Pa}]$ & $1.4 \times 10^{8}$ & $9.01 \times 10^{5}$ & $1.49 \times 10^{10}$ \\
$\rho\left[\mathrm{kg} \cdot \mathrm{m}^{-3}\right]$ & $3.95 \times 10^{3}$ & $9 \times 10^{2}$ & $11.6 \times 10^{3}$ \\
\hline \hline
\end{tabular}

a Oxide of Oxide of Aluminium $\left(\mathrm{Al}_{2} \mathrm{O}_{3}\right)$.

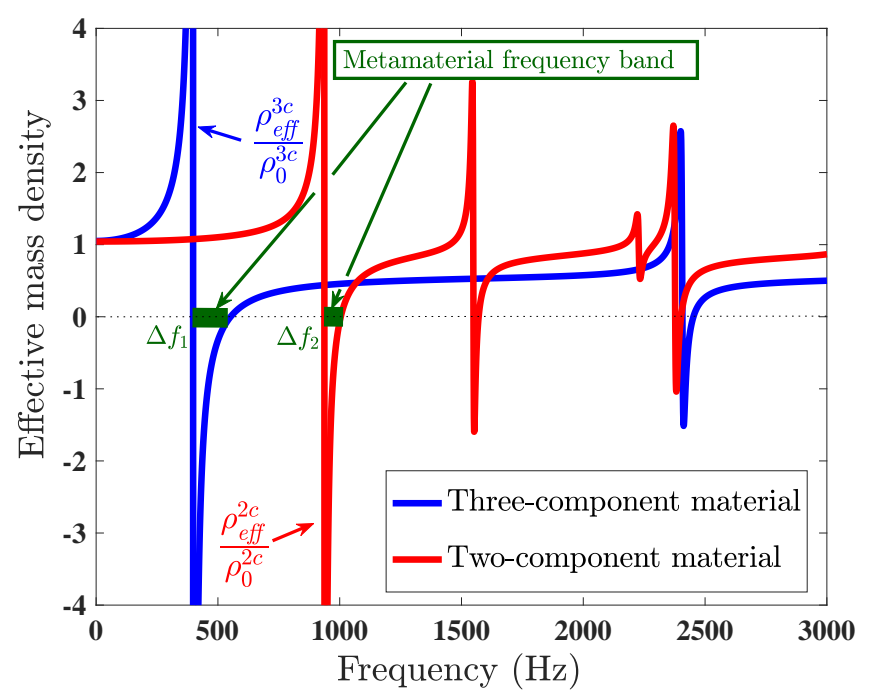

FIG. 2. (Color online) Normalized effective mass density related to two-component and threecomponent materials with spherical inclusions. First metamaterial bands $\Delta f_{1}$ and $\Delta f_{2}$, where the effective mass densities become negative, are shown by green lines for both types of metasolids.

to compare their metamaterial characteristics. Then, we proceed to perform a parametric study to analyze metamaterial properties in terms of geometrical configuration of this class of metasolids.

Fig. 2 shows the effective density of a twocomponent metasolid and a three-component one with spherical inclusions. The structural properties of the two-component metasolid, composed of a stiff matrix and an elastic inclusion, are presented in Table I. The components of this material are chosen as in Ref. ${ }^{14}$. Obviously, these properties are chosen such that they satisfy the conditions that were explained in Sec. II, allow- 
TABLE II. Expressions of the resonance frequencies and their frequency bandwidths. The quantity $\bar{f}_{0}$ is defined as $\bar{f}_{0}=(1 / 2 \pi) \sqrt{\mu_{2} /\left(\rho_{3} b^{2}\right)}$.

\begin{tabular}{l}
\hline Geometry \\
Resonance frequency $\left(f_{0}\right) \quad \bar{f}_{0}\left\{\frac{8(1-\nu)(3-4 \nu) \gamma^{2}}{(3-4 \nu)^{2} \ln \gamma-\frac{\gamma^{2}-1}{\gamma^{2}+1}}\right\}^{1 / 2} \quad \bar{f}_{0}\left\{\frac{18(1-\nu)(2-3 \nu) \gamma^{2}}{(5-6 \nu)(2-3 \nu)\left(\frac{\gamma-1}{\gamma}\right)-\frac{5}{4} \frac{\left(\gamma^{2}-1\right)^{2}}{\gamma^{5}-1}}\right\}^{1 / 2}$ \\
Frequency bandwidth $(\Delta f) \quad f_{0}\left\{\sqrt{1+\left(\frac{\rho_{3}}{\rho_{1}}\right)\left(\frac{1-\phi_{1}}{\phi_{1} \gamma^{2}}\right)}-1\right\} \quad f_{0}\left\{\sqrt{1+\left(\frac{\rho_{3}}{\rho_{1}}\right)\left(\frac{1-\phi_{1}}{\phi_{1} \gamma^{3}}\right)}-1\right\}$ \\
\hline \hline
\end{tabular}

ing us to consider the matrix as a rigid body. The length of the unit cell (see Fig. 1a) $\ell=4.06 \mathrm{~cm}$, the radius $b$ of the spherical inclusion is $2 \mathrm{~cm}$ and its volume fraction is $50 \%$. Regarding the threecomponent material, the same material properties are chosen as those for the two-component one, and a lead core (Table I) of radius $a=1.32$ cm with a volume fraction of $\phi_{3}=14.2 \%$ is added at the center of the unit cell (Fig. 1a). Effective mass densities for the three-component and the two-component materials are calculated via Eqs. (13) and (15), respectively. The first resonance frequency for the two-component material is at $937 \mathrm{~Hz}$, whereas that produced by the threecomponent material is at $367 \mathrm{~Hz}$. Thus, it can be seen that while keeping the same geometrical parameters in terms of the size of the unit cell and the inclusion, adding a rigid mass at the center of the unit cell could make the metamaterial effect, that means the emergence of the negative mass density, occur at significantly lower frequency. Indeed, in three-component metasolid, this particular local-resonance effect is due to the motion of the rigid core. It is worth noting that, this is not necessarily a general result. Indeed, when $\gamma$ is decreased, according to the mass-spring model $^{3}$, both the mass and the stiffness of the spring (analogous to the cladding) are decreased. As the mass in the mass-spring model decreases with $\gamma$ faster than the stiffness, the resonance frequency associated with the motion of the rigid mass is shifted to higher frequencies [see the expression of the resonance frequency for spherical inclusions in Table II and Fig. 8 in Ref. ${ }^{3}$; while the resonance frequency related to the motion inside the cladding is relatively stable.
Given the size of the unit cell and the inclusion, the rigid mass has been placed inside the inclusion so that the first resonance frequency, and thereby the frequency of the first metamaterial effect, would be minimized. To do so, the massspring model and the explicit expression of the resonance frequency within this model in Table II Ref. $^{3}$ ) are used to determine the form factor $\gamma$ that, here, is found to be 1.52 .

It is also to be noted that within the frequency band shown in Fig. 2, there are more resonance effects associated with the twocomponent metasolid compared with the threecomponent one; all, evidently, are caused by the motion inside the homogeneous soft inclusion. It has been previously explaind that the second resonance effect at about $2407 \mathrm{~Hz}$ for the threecomponent material occurs inside the cladding ${ }^{3}$. The greater number of resonance effects with the two-component metasolid is simply due to the fact that the volume of the soft elastic material, capable of generating several resonances at low frequencies, in such composite is larger than that involved in three-component material. Remarkably, it can be further observed that not only the first local resonance of the three-component material appears at lower frequency compared with that of the two-component material, but also its metamaterial frequency band $\Delta f$ is larger (see Fig. $2, \Delta f_{1}=132 \mathrm{~Hz}, \Delta f_{2}=53 \mathrm{~Hz}$ ).

These results show the relevance of introducing an additional tuning parameter, that is the volume fraction of the biphasic inclusion, in order to optimize the homogenized properties. Let us see through Fig. 3 how far we can pursue optimal tunability. The curve in Fig. 3 shows the evolution of the metamaterial frequency band- 


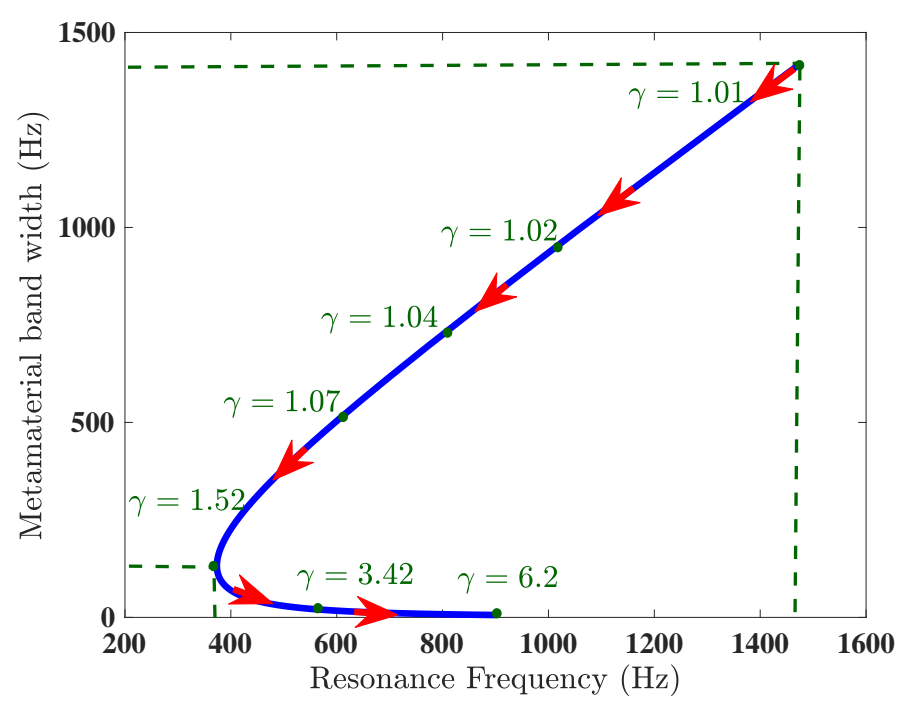

FIG. 3. (Color online) Evolution of the metamaterial bandwidth with the resonance frequency. The curve is parameterized by the form factor $\gamma$. The red arrows indicate the direction of increasing $\gamma$.

width $\Delta f$ as a function of the resonance frequency $f_{0}$ of the rigid body mode for the threecomponent metasolid; with $f_{0}$ being the lower limit of the frequency band where the effective mass density is negative. The curve is parameterized by the form factor $\gamma$, which evolves increasingly by starting from 1.01 (top right) to 6.2 (bottom right). The starting value for $\gamma$, clearly, corresponds to the configuration where the cladding is filled by the lead core and the inclusion is almost entirely rigid. It is seen that for a given choice of inclusion components and its volume fraction in a given matrix, it is not possible to achive, at the same time, both the lowest resonance frequency and the maximum of bandwidth. Indeed, if the aim is to have the resonance frequency as low as possible, then there is an optimal $\gamma$ value, $\gamma=1.52$; but in this case, the bandwidth is far from its largest possible value. To have a larger bandwidth, the size of the rigid mass in the inclusion, should be increased, accepting that the resonance frequency be shifted upwards in the spectrum. Furthermore, as $\gamma$ increases up to the value 1.52 ; with a fixed volume fraction of the inclusion, the size of the rigid core is decreased, and thereby the metasolid becomes lighter. In this case, both the resonance frequencies and their associated metamaterial bandwidths decrease. For large values
TABLE III. Material properties of the threecomponent unit-cell (Ref. ${ }^{15}$ ).

\begin{tabular}{cccc}
\hline \hline Material & $\begin{array}{c}\mathrm{Al} \\
(\text { matrix })\end{array}$ & $\begin{array}{c}\text { PVC } \\
(\text { cladding })\end{array}$ & $\begin{array}{c}\text { Lead } \\
(\text { core })\end{array}$ \\
$\lambda[\mathrm{Pa}]$ & $5.74 \times 10^{10}$ & $1.6 \times 10^{5}$ & $4.23 \times 10^{10}$ \\
$\mu[\mathrm{Pa}]$ & $2.7 \times 10^{10}$ & $4 \times 10^{7}$ & $1.49 \times 10^{10}$ \\
$\rho\left[\mathrm{kg} \cdot \mathrm{m}^{-3}\right]$ & $2.5 \times 10^{3}$ & $1.5 \times 10^{3}$ & $11.6 \times 10^{3}$ \\
\hline \hline
\end{tabular}

of $\gamma$ and in the limit $\gamma \gg 1$, the inclusion consists of only elastic material and the metasolid produces low-frequency negative mass densities with very small bandwidths; in accord with what was seen earlier through Fig. 2 with the red curve. These observations imply a compromise to be found depending on the type of application of such metasolids; but this predictive tool allows targeting the values in question without heavy calculations, as it is based on the massspring model that was validated in our previous paper $^{3}$.

\section{DESIGN OF OPTIMAL STRUCTURES}

The observations and analysis of the previous section leads to summarize a methodology describing the inverse problem (downscaling) to determine the optimal material parameters at the micro level. At the macro level, via solving the forward problem (upscaling), the change in the performance of the optimal metasolids is then shown compared to some typical cases that are reported in the literature. This suggests a choice of material components and certain geometrical parameters at micro level. It is shown that by keeping the initial material choices, the tunability and performance of the metasolids can be significantly improved with respect to the resonance frequency and width of the frequency band for the metamaterial effect.

The methodology is based on the links established between data at micro level of the structure and those at the macro level where the material is considered as homogeneous. The microlevel data are the geometrical ones; 3 independent parameters among $\left\{a, b, \ell, \gamma, \phi_{i}\right\}$ and material properties $\left\{\right.$ densities $\rho_{i}$, moduli of elasticity $\lambda_{i}$, $\left.\mu_{i}\right\}, i=1,2,3$, i.e. three constants per phase that should, indeed, satisfy the conditions of contrast 
reported within the first paragraphs of Sec. II). The macro-level data of interest are contained in the evolution of the effective density $\rho_{\text {eff }}$ with $\omega$; in particular the resonance frequency $f_{0}$ and metamaterial frequency bandwidth $\Delta f$, which will be investigated here.

In order to compare the performance of the two-component ${ }^{14,15}$ versus three-component metasolids, or simply optimize the behavior of the three-component effective metasolids ${ }^{2}$, the micro parameters should be determined in accordance with a targeted macro-structural behavior. This raises a downscaling or inverse problem that is quite difficult to solve by the continuous elastodynamic description that was presented in Sec. II B. The simplified mass-spring model $^{3}$ is therefore used to circumvent this difficulty, adopting the following algorithm:

1. For the homogenized medium, criteria are imposed on the selected target quantities (here $f_{0}$ and $\Delta f$ from $\left.\rho_{\text {eff }}(\omega) / \rho_{0}\right)$.

2. These target quantities are imported to the associated homogeneous medium that is homogenized based on the mass-spring model within which we have $\frac{m_{\text {eff }}(\omega)}{m_{0}}=\frac{1-\left(\omega / \omega_{c}\right)^{2}}{1-\left(\omega / \omega_{0}\right)^{2}}$, with $\omega_{c}=\omega_{0}+\Delta \omega=\omega_{0} \sqrt{1+m_{3} / m_{1}}, \omega_{0}$ being the angular frequency of the first local resonance, $\Delta \omega$ the bandwidth of the metamaterial effect, $m_{1}$ (resp. $m_{3}$ ) the mass of the rigid inclusion (resp. matrix), and $m_{0} \equiv m_{1}+m_{2}$ the static limit of the effective mass $m_{\text {eff }}(\omega)$. It is through this relation that the link between the two homogeneous media, homogenized by the elastodynamic model (Sec. II B) and that by the mass-spring model ${ }^{3}$, is established. Indeed, $\left(m_{\text {eff }}(\omega) / m_{0}\right)_{\text {mass-spring }} \simeq$ $\left(\rho_{\text {eff }}(\omega) / \rho_{0}\right)_{\text {elastodynamic }}$ at low frequencies including the first resonance frequency ${ }^{3}$, which makes it possible from now on to envisage solving the inverse problem in a much easier way.

3. Downscaling is performed then from the effective medium homogenized by the massspring model; for example, searching $\gamma$ which minimizes $f_{0}$. The minimization of $f_{0}$ with respect to $\gamma$ is carried out through the simple explicit relation given in the first row of Table II (search for a minimum of a function of a single variable).

4. Once the micro parameters thus determined (by the mass-spring model), an iterative process is initiated to obtain the properties of the effective medium homogenized in the sense of elastodynamic model by using Eqs. (12) and (13) to find $f_{0}$ and $\Delta f$ according to the elastodynamic homogenization scheme. In fact, since the inverse problem in the elastodynamic model is much complicated to solve, we thus simply calculate several solutions iterating the forward elastodynamic problem in the neighborhood of a 'good' initial value provided by the solution of the inverse problem based on the mass-spring model. The mass-spring model has precision issues, especially for predicting $\Delta f$; which requires returning to the macroscale via the elastodynamic model to examine the effective behavior imposed by the choice of the micro-structure, such as $\gamma$ minimizing $f_{0}$. In what follows in the paper, this step is operated in two ways:

4 -a. The inverse problem is to find $\gamma$ as a micro parameter minimizing $f_{0}$ as a macro target quantity; all other parameters being fixed. An example is presented in Fig. 2. In this case, one single iteration from this value of $\gamma$ is enough to obtain the desired $f_{0}$ based on the elastodynamic model. It is shown that a three-component material is more efficient than a two-component material, since with the same material components and geometrical parameters, a much lower $f_{0}$ can be achieved.

4-b. The target macro quantities are $f_{0}$ and $\Delta f$, while $\gamma$ is the only micro parameter. A compromise for a relatively low $f_{0}$ and a relatively large $\Delta f$ is searched. Several iterations are performed from the initial $\gamma$ and by decreasing it (as suggested by the parametric study of the type presented in Fig. 3). Fixing thresholds in advance, the iterations are stopped, when a sufficiently large $\Delta f$ and a $f_{0}$ that is not too high are reached, .

Comparing two-component and threecomponent materials, a first example was discussed in Sec. III in which material components (Table I), the length of the periodic unit cell $\ell$ and the size of the mono-phasic spherical inclusion represented by $b$ have been extracted from Ref. ${ }^{14}$. Based on these inputs, the threecomponent material is made by adding a lead core at the center of the inclusion. In order to have the minimum resonance frequency for the new three-component material (see step 4-a of the above algorithm), the inverse problem leads to the micro-parameter $\gamma=1.52$. Thus, initial values in two-component metasolid are improved from $\left(f_{0}, \Delta f\right)=(937,25) \mathrm{Hz}$ to the values in the 
TABLE IV. Material properties of the threecomponent unit-cell (Ref. ${ }^{2}$ ).

\begin{tabular}{cccc}
\hline \hline Material & $\begin{array}{c}\text { Epoxy } \\
(\text { matrix })\end{array}$ & $\begin{array}{c}\text { Silicone } \\
(\text { cladding })\end{array}$ & $\begin{array}{c}\text { Lead } \\
(\text { core })\end{array}$ \\
$\lambda[\mathrm{Pa}]$ & $4.43 \times 10^{9}$ & $6 \times 10^{5}$ & $4.23 \times 10^{10}$ \\
$\mu[\mathrm{Pa}]$ & $1.59 \times 10^{9}$ & $4 \times 10^{4}$ & $1.49 \times 10^{10}$ \\
$\rho\left[\mathrm{kg} \cdot \mathrm{m}^{-3}\right]$ & $1.18 \times 10^{3}$ & $1.3 \times 10^{3}$ & $11.6 \times 10^{3}$ \\
\hline \hline
\end{tabular}

optimized case for the three-component one: $\left(f_{0}, \Delta f\right)=(367,64) \mathrm{Hz}$. As such, $f_{0}$ is divided by almost 3 and $\Delta f$ is multiplied by more than 2. The bandwidth becomes larger, at the end, without being considered as an optimization parameter in this procedure.

The same optimization procedure is applied with input quantities from Ref. ${ }^{15}$ for a twocomponent metasolid. Material components are reported in Table III, with $\ell=1 \mathrm{~mm}$ and $b=$ $0.415 \mathrm{~mm}$, i.e. volume fraction of $54 \%$ for, this time, the cylindrical inclusions. As before, to construct the three-component metasolid we add a lead core (Table III) at the center of the inclusion. Comparison of the two-component and the optimized three-component materials makes it possible to change the values of the resonance frequency and metamaterial bandwidth to $\left(f_{0}, \Delta f\right)=(129,75) \mathrm{kHz}$ from those in Ref. ${ }^{15}$ $\left(f_{0}, \Delta f\right)=(268,23) \mathrm{kHz}$. Again, it is seen that the resonance frequency is decreased, and the width of the metamaterial-effect frequency band is increased.

The third example relates to the threecomponent metasolid discussed in Ref. ${ }^{2}$ with material properties shown in Table IV. The geometrical configuration for spherical inclusions are $\ell=15.5 \mathrm{~mm}, b=7.5 \mathrm{~mm}$, (volume fraction of $40 \%$ ) and for cylindrical inclusions, $\ell=21 \mathrm{~mm}$ and $b=7.5 \mathrm{~mm}$ (volume fraction of $47 \%$ ). With these material and geometrical parameters, for spherical inclusion, $\left(f_{0}, \Delta f\right)=(374,230) \mathrm{Hz}$, the form factor being as $\gamma=1.5$, while the optimized value of the form factor $\gamma=2.04$ minimizing the resonance frequency, leads to $\left(f_{0}, \Delta f\right)=(337,83)$ Hz. Hence, with the choices in Ref. ${ }^{2}$, a relative increase in resonant frequency of $11 \%$ but also a relative increase in bandwidth of $177 \%$ are observed. Comparison with Ref. ${ }^{2}$ for the case of cylindrical inclusions, where $\gamma$ remains the same at 1.5 and $\left(f_{0}, \Delta f\right)=(356,280) \mathrm{Hz}$, using the same optimization criteria as before, results in $\left(f_{0}, \Delta f\right)=(312,105) \mathrm{Hz}$. That is, according to the choices in Ref. ${ }^{2}$, there is a relative increase of $14.1 \%$ in the resonance frequency with respect to the optimized material, and at the same time, a relative increase of $167 \%$ in the bandwidth. It can be noted that the form factor found as $\gamma=2.04$ allows the resonance frequency to be lowered compared to that corresponding to the initial three-component configuration. However, it can be seen that increasing the resonance frequency slightly higher than its possible minimum value corresponds to a configuration $(\gamma=1.5)$ that allows the bandwidth to be widened significantly. This behavior with $\gamma$ and $f_{0}$ could be noted through the example illustrated in Fig. 3 in the previous section.

This suggests exploiting a new optimization criteria (see step 4-b of the algorithm) by setting a compromise for low resonance frequency and wide bandwidth based on the numerical values reported in Ref. ${ }^{2}$. The new inverse problem can be thus defined as finding $\gamma$ such that $f_{0}$ is $11 \%$ for cylindrical and $14.1 \%$ for spherical inclusions, higher than its minimum value, while increasing the metamaterial bandwidth. If the first two examples, i.e. two-component materials from Ref. ${ }^{14}$ and Ref. ${ }^{15}$, are reconsidered with the new optimization criteria, the inverse problem generates a form factor $\gamma=1.23$ (instead of 1.52) regarding the optimized tree-component version of the two-component metasolid discussed in Ref. ${ }^{14}$ (Table I), which, by using the direct elastodynamic equations, yields $\left(f_{0}, \Delta f\right)=(408,123) \mathrm{Hz}$. That means a relative increase of $f_{0}$ of $11.2 \%$ and simultaneously a relative increase of $\Delta f$ of about $92 \%$ with respect to the values produced by the initial optimization. Similarly, with the metasolid from Ref. ${ }^{15}$ (Table III), the new inverse problem provides $\gamma=1.31$ (instead of 1.79) to achieve then the values of $\left(f_{0}, \Delta f\right)=(147,145)$ $\mathrm{kHz}$ for the three-component composite, which means a relative increase of about $14 \%$ for $f_{0}$ and that of $91 \%$ for $\Delta f$ with respect to the values that were obtained based on minimization of the resonance frequency.

\section{CONCLUDING REMARKS}

Further analyses were performed on metasolid properties of composite materials with structural units made of a stiff matrix with ei- 
ther an elastic inclusion, or an elastic inclusion embedding a rigid mass inside itself. The essence of this paper concerned the comparison of the homogenised dynamics of a soft monophasic inclusion, that is statically homogeneous, and a biphasic inclusion constituted of a rigid core coated by an elastic material; each of which is embedded in a stiff matrix. The effectivemedium parameter that describes the macroscopic behavior of these structures is the effective mass density, which becomes negative near the resonance frequency of the unit cell.

Thus, it was shown by an analytic model and parametric analysis that bi-inclusion threecomponent materials could provide a scope of material dynamics much larger compared with that of the mono-inclusion two-component materials. It was clarified that local-resonance frequencies are relatively high with two-component materials, because even in the case where the elasticity coefficients related to the matrix and the inclusion are sufficiently contrasted, the contrast may not exist for the mass densities; and vice versa. With such materials, it is indeed very difficult to find, in practice, a low rigidity and, at the same time, a high mass density for the elastic material used as inclusion inside the stiff matrix. It was shown that this could be feasible by dissociating these two material properties, i.e. softness for the cladding and mass density for the coated rigid core, with a three-component material whose inclusion is composed of two phases.

Additionally, in order to increase the bandwidth of metamaterial effect where the effective mass density is negative, a large volume fraction is required for the vibrating part in opposition of phase with the matrix, which is easier to implement with a three-component material. Also, a parametric study was performed to analyze the interplay between the form factor-specifying the volume fraction of the rigid mass and cladding, the metamaterial-effect bandwidth and the resonance frequency- which ultimately allows, via an inverse problem, the design of metasolid with desired properties.

Conclusively, adding a rigid mass inside the elastic inclusion of the two-component material will significantly increase the tunability of the composite material, via enlarging the domain of inclusion dynamic properties, and widening remarkably the range of values of material effective mass density. This is interesting for various applications such as acoustic and vibration insulation. The analysis employed in this paper can be straightforwardly generalized to treat polyinclusion multi-component materials.

\section{ACKNOWLEDGMENTS}

This work has benefited from a French government Grant managed by ANR within the framework of the National Program Investments for the Future ANR-11-LABX-0022-01 (LabEx MMCD project).

\section{APPENDIX A: SOLUTIONS TO HELMHOLTZ EQUA- TIONS}

Solving the resulting Helmholtz equations (5) and regarding the mirror symmetry about the $x$-axis $(\Phi$ is an odd function and $\Psi$ is an even function of $\theta$ ), we have

$$
\begin{aligned}
& \Phi(r, \theta)=\sum_{n=0}^{\infty}\left[A_{n} \mathrm{~J}_{n}(h r)+B_{n} \mathrm{Y}_{n}(h r)\right] \cos (n \theta) \\
& \Psi(r, \theta)=\sum_{n=0}^{\infty}\left[E_{n} \mathrm{~J}_{n}(\kappa r)+F_{n} \mathrm{Y}_{n}(\kappa r)\right] \sin (n \theta) \boldsymbol{e}_{z}
\end{aligned}
$$

for the $2 \mathrm{D}$ system, where $\mathrm{J}_{n}\left(r e s p . \mathrm{Y}_{n}\right)$ stands for the Bessel function of the first (resp. second) kind. The boundary conditions (6) select only the terms corresponding to $n=1$ in the above expansions:

$$
\begin{aligned}
& \Phi(r, \theta)=\left[A_{1} \mathrm{~J}_{1}(h r)+B_{1} \mathrm{Y}_{1}(h r)\right] \cos \theta \\
& \boldsymbol{\Psi}(r, \theta)=\left[E_{1} \mathrm{~J}_{1}(\kappa r)+F_{1} \mathrm{Y}_{1}(\kappa r)\right] \sin \theta \boldsymbol{e}_{z}
\end{aligned}
$$

By following the same procedure including the boundary conditions (7) we obtain the expression of the potentials for the 3D system:

$$
\begin{aligned}
\Phi(R, \varphi) & =\sum_{n=0}^{\infty}\left[a_{n} \mathrm{j}_{n}(h R)+b_{n} \mathrm{y}_{n}(h R)\right] P_{n}^{0}(\cos \varphi) \\
& =\left[a_{1} \mathrm{j}_{1}(h R)+b_{1} \mathrm{y}_{1}(h R)\right] \cos \varphi \\
\Psi(R, \varphi) & =-\sum_{n=1}^{\infty}\left[e_{n} \mathrm{j}_{n}(\kappa R)+f_{n} \mathrm{y}_{n}(\kappa R)\right] P_{n}^{1}(\cos \varphi) \boldsymbol{e}_{\theta} \\
& =\left[e_{1} \mathrm{j}_{1}(\kappa R)+f_{1} \mathrm{y}_{1}(\kappa R)\right] \sin \varphi \boldsymbol{e}_{\theta}
\end{aligned}
$$

where $\mathrm{j}_{n}$ (resp. $\mathrm{y}_{n}$ ) stands for the spherical Bessel functions of the first (resp. second) kind, and $P_{n}^{k}$ are the associated Legendre polynomials of order $k$. 
In these solutions, $A_{1}, B_{1}, E_{1}, F_{1}, a_{1}, b_{1}, e_{1}$, and $f_{1}$, are unknown constants to be determined by using the boundary relations at the interfaces of each two phases in the structural unit.

\section{APPENDIX B: ALGEBRAIC SYSTEMS}

The following linear algebraic systems allow the determination of the constants and are involved in the equations (A2) and (A3), respectively. For the 2D system, we have

$$
\underbrace{\left(\begin{array}{cccc}
h a \mathrm{~J}_{0}(h a) & h a \mathrm{Y}_{0}(h a) & \kappa a \mathrm{~J}_{0}(\kappa a) & \kappa a \mathrm{Y}_{0}(\kappa a) \\
h a \mathrm{~J}_{2}(h a) & h a \mathrm{Y}_{2}(h a) & -\kappa a \mathrm{~J}_{2}(\kappa a) & -\kappa a \mathrm{Y}_{2}(\kappa a) \\
h b \mathrm{~J}_{0}(h b) & h b \mathrm{Y}_{0}(h b) & \kappa b \mathrm{~J}_{0}(\kappa b) & \kappa b \mathrm{Y}_{0}(\kappa b) \\
h b \mathrm{~J}_{2}(h b) & h b \mathrm{Y}_{2}(h b) & -\kappa b \mathrm{~J}_{2}(\kappa b) & -\kappa b \mathrm{Y}_{2}(\kappa b)
\end{array}\right)}_{D}\left(\begin{array}{c}
A_{1} \\
B_{1} \\
E_{1} \\
F_{1}
\end{array}\right)=\left(\begin{array}{c}
2 a U_{a} \\
0 \\
2 b U_{b} \\
0
\end{array}\right)
$$

and for the 3D system:

$$
\underbrace{\left(\begin{array}{cccc}
h a \mathrm{j}_{0}(h a) & h a \mathrm{y}_{0}(h a) & 2 \kappa a \mathrm{j}_{0}(\kappa a) & 2 \kappa a \mathrm{y}_{0}(\kappa a) \\
h a \mathrm{j}_{2}(h a) & h a \mathrm{y}_{2}(h a) & -\kappa a \mathrm{j}_{2}(\kappa a) & -\kappa a \mathrm{y}_{2}(\kappa a) \\
h b \mathrm{j}_{0}(h b) & h b \mathrm{y}_{0}(h b) & 2 \kappa b \mathrm{j}_{0}(\kappa b) & 2 \kappa b \mathrm{y}_{0}(\kappa b) \\
h b \mathrm{j}_{2}(h b) & h b \mathrm{y}_{2}(h b) & -\kappa b \mathrm{j}_{2}(\kappa b) & -\kappa b \mathrm{y}_{2}(\kappa b)
\end{array}\right)}_{d}\left(\begin{array}{c}
a_{1} \\
b_{1} \\
e_{1} \\
f_{1}
\end{array}\right)=\left(\begin{array}{c}
3 a U_{a} \\
0 \\
3 b U_{b} \\
0
\end{array}\right)
$$

${ }^{1}$ Z. Liu, X. Zhang, Y. Mao, Y. Y. Zhu, Z. Yang, C. T. Chan, and P. Sheng, "Locally resonant sonic materials," Science 289, 1734 (2000).

${ }^{2}$ Z. Liu, C. T. Chan, and P. Sheng, "Analytic model of photonic crystals with local resonances," Phys. Rev. B 71, 014103 (2005).

${ }^{3}$ E. Favier, N. Nemati, C. Perrot, and Q. C. He, "Generalized analytic model for rotational and anisotropic metasolids," J. Phys. Comm. 2, 035035 (2018).

${ }^{4}$ M. M. Sigalas and E. N. Economou, "Elastic and acoustic wave band structure," J. Sound Vib. 158, 377-382 (1992).

${ }^{5}$ M. S. Kushwaha, P. Halevi, L. Dobrzynski, and B. Djafari-Rouhani, "Acoustic band structure of periodic elastic composites," Phys. Rev. Lett. 71, 2022 (1993).

${ }^{6}$ D. Torrent, Y. Pennec, and B. Djafari-Rouhani, "Resonant and nonlocal properties of phononic metasolids," Phys. Rev. B 92, 174110 (2015).

${ }^{7}$ N. Nemati, A. Kumar, D. Lafarge, and N. X. Fang, "Nonlocal description of sound propagation through an array of Helmholtz resonators," C. R. Mecanique, 343, 656-669 (2015).

${ }^{8}$ N. Nemati, Y. K. Lee, D. Lafarge, A. Duclos, and N. Fang, "Nonlocal dynamics of dissipative phononic fluids," Phys. Rev. B 95, 224304 (2017).

${ }^{9}$ G. M. Milton and J. R. Willis, "On modifications of Newton's second law and linear continuum elastodynamics," Proc. R. Soc. A 463, 855 (2007).
${ }^{10}$ Y. Wu, Y. Lai, and Z. Q. Zhang, "Effective medium theory for elastic metamaterials in two dimensions," Phys. Rev. B 76, 205313 (2007).

${ }^{11}$ R. C. McPhedran and A. B. Movchan, "The Rayleigh multipole method for linear elasticity," J. Mech. Phys. Solids 42, 711-727 (1994).

${ }^{12} \mathrm{~J} . \mathrm{Li}$ and C. Chan, "Double-negative acoustic metamaterial," Phys. Rev. E 70, 055602 (2004).

${ }^{13}$ M. Yang, G. Ma, Y. Wu, Z. Yang, and P. Sheng, "Homogenization scheme for acoustic metamaterials," Phys. Rev. B 89, 064309 (2014).

${ }^{14} \mathrm{G}$. Bonnet and V. Monchiet, "Dynamic mass density of resonant metamaterials with homogeneous inclusions," J. Acoust. Soc. Am. 142, 890 (2017).

${ }^{15}$ J. J. Marigo, A. Maurel, Second order homogenization of subwavelength stratified media including finite size effect, SIAM J. Appl. Math., 77(2), 721-743 (2017).

${ }^{16} \mathrm{H}$. H. Huang and C. T. Sun, "Wave attenuation mechanism in an acoustic metamaterial with negative effective mass density," New J. Phy. 11, 013003 (2009).

${ }^{17} \mathrm{G}$. Bonnet and V. Monchiet, "Low frequency locally resonant metamaterials containing composite inclusions," J. Acoust. Soc. Am. 137, 3263 (2015).

${ }^{18}$ J. L. Auriault and C. Boutin, "Long wavelength innerresonance cut-off frequencies in elastic composite materials," Int. J. Solids Struct. 49(23-24), 3269-3281 (2012). 
${ }^{19}$ X. J. L. Auriault and G. Bonnet, "Dynamique des composites élastiques periodiques" ("Dynamics of elastic periodic composites"), Arch. Mech. 37(4), 269-284 (1985).

${ }^{20}$ Y. J. L. Auriault, "Acoustics of heterogeneous media: Macroscopic behavior by homogenization," Cur- rent Topics Acoust. Res. 1, 63-90 (1994). 